EEST1 NSV TEADUSTE AKADEEMIA TOIMETISED. 29. KOIDE FUUSIKA * MATEMAATIKA. 1980, NR. 4

ИЗВЕСТИЯ АКАДЕМИИ НАУК ЭСТОНСКОИ ССР. ТОМ 29 ФИЗИҚА * МАТЕМАТИҚА. 1980, № 4

\title{
АНАЛИЗ КВАНТОВОКИНЕТИЧЕСКИХ УРАВНЕНИЙ БЛОКА
}

\author{
(Представил Э. Липпмаа)
}

Выведенные Блоком квантовокинетические уравнения $\left[{ }^{1}\right]$ широко применяются в спектроскопии ядерного магнитного резонанса (ЯМР) жидкостей. Сходные уравнения оправдали себя также в других областях радиоспектроскопии и в квантовой электронике $\left[{ }^{2-4}\right]$.

Более систематический анализ физического содержания уравнений Блока $\left[{ }^{1}\right]$ требует подходящего математического аппарата. В [ $\left.{ }^{5}\right]$ указывалось, что таковым мог бы служить аппарат теории групп $\mathbf{G L}\left(n^{2}, R\right)$ и их алгебр Ли. В данной работе этот вопрос изучается на примере $n$-уровневых спиновых систем, находящихся в условиях слабого возбуждения и слабой релаксации. Устанавливаются общие ограничения, налагаемые на пропагаторы уравнениями Блока. Общие свойства этих уравнений выражены на языке пропагаторов.

Здесь уравнения Блока рассмотрены с феноменологической точки зрения. Показано, что вид этих уравнений почти полностью определяется такими требованиями общего характера, как свойства оператора плотности, линейность кинетики, свойства симметрии релаксационного процесса, больцмановский закон равновесного состояния и существование шредингеровской поддинамики, описывающей предельный случай обратимых процессов. Теория относится к больцмановскому ансамблю n-уровневых квантовых систем без указания на конкретную природу системы и ее взаимодействий. Этим объясняется применимость теории Блока вне рамок ЯМР.

\section{Квантовокинетическое уравнение Блока}

Временная эволюция оператора плотности $P(t) n$-уровневой спиновой системы описывается $n^{2}$ линейными, однородными дифференциальными уравнениями [ $\left.{ }^{1}\right]$. Если рассматривать $P(t)$ в качестве вектора $n^{2}$-мерного унитарного пространства $\mathbf{O}\left[{ }^{6}\right]$ и ввести действующие в $\mathbf{O}$ супероператоры $\left[{ }^{6,7}\right]$, то уравнениям Блока $\left[{ }^{1}\right]$ можно придать следующий компактный вид

$$
\frac{d P}{d t}=(\mathfrak{S}(t)+(\mathfrak{H}) P
$$

Входящий в уравнение (1) антиэрмитовый супероператор $\mathfrak{g}(t)$ (супергамильтониан) состоит из постоянной и переменной частей

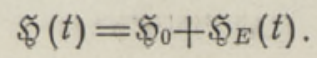


$\S_{E}(t)$ описывает взаимодействие спиновой системы с внешними (классическими) переменными полями, \$o же ответствен за взаимодействие с постоянными полями и за усредненное взаимодействие с молекулярным окружением спиновой системы (с термостатом) $\left[{ }^{1,8}\right]$. Если гамильтонианом спиновой системы является эрмитовый оператор

$$
H(t)=H_{0}+H_{E}(t) \in \mathbf{0},
$$

то соответствующий супергамильтониан вычисляется по уравнению

$$
\xi(t) P=-i[H(t), P] .
$$

Супероператор релаксации б5 характеризует стохастические (тепловые) взаимодействия между спиновой системой и термостатом [ $\left.{ }^{8}\right]$. Конкретный вид супероператора релаксации раскрывается ниже. Будет показано, что \&) в значительной мере предопределяется следующими требованиями:

$$
\begin{aligned}
& \mathscr{S}(t)+\mathscr{G} \in \mathbf{g l}\left(n^{2}, R\right) \text {, } \\
& \operatorname{tr} P(t)=1, \\
& 0<(P(t), P(t)) \leqslant 1 \text {, }
\end{aligned}
$$

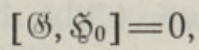

$$
\begin{aligned}
& P^{0}=(1 / Z) \exp \left(-\hbar H_{0} / k T\right) \text {, } \\
& Z=\operatorname{tr} \exp \left(-\hbar H_{0} / k T\right) \text {. }
\end{aligned}
$$

Требования $(5)-(7)$ вытекают из основных свойств оператора плотности. В условиях релаксации $\left(H_{E}(t)=0\right)$ спиновой ансамбль, движение которого подчиняется уравнениям (1), (5)-(7), будет стремиться к равновесному состоянию $P^{0}$, которое должно быть, согласно (9) и (10), больцмановским распределением. С этим согласуется требование (8).

$A$ - бази с прост р анств а О. Базис, составленный из собственных векторов $A_{m k}(m, k=1,2, \ldots, n)$ супероператора $\mathfrak{S}_{0}$, назовем $A$-базисом [ $\left.{ }^{6}\right]$. Если

$$
H_{0}|m\rangle=\omega_{m}^{0}|m\rangle \quad(m=1,2, \ldots, n),
$$

то базису $|m\rangle \in \mathbf{C}$ (пространство чистых квантовых состояний) соответствует ортонормированный $A$-базис:

$$
\mathfrak{S}_{0} A_{m k}=\omega_{m k}^{0} A_{m k},
$$

где

$$
\stackrel{0}{\omega_{m k}}=\stackrel{0}{\omega_{m}}-\stackrel{0}{\omega_{k}}
$$

- т. н. резонансная частота перехода $m \rightarrow k$.

Условия применимости теории Блока. Будем различать общие условия применимости теории Блока $\left[{ }^{1}\right]$ и более специфические дополнительные условия, принятые в данной работе.

Общими условиями являются:

$$
1 / \tau_{c}, k T / \hbar \gg\left|\breve{S}_{E}\right|, \mid \text { |S } \mid \text {. }
$$

В неравенствах (14) знак абсолютного значения указывает на поря- 
док величины матричных элементов вписанного супероператора. $\tau_{c}$ - время корреляции стохастических взаимодействий $\left[{ }^{8}\right]$.

В качестве специальных условий слабого возбуждения и слабой релаксации принимаем:

$$
\left|\omega_{m n}^{0}\right|,\left|\omega_{m n}^{0}-\omega_{k l}^{0}\right| \gg\left|\breve{S}_{E}\right|, \mid \text { (5) }
$$

при $m \neq n \neq k \neq l$. Итак, уровни энергии $\omega_{m}{ }^{0}$ невырождены и равные резонансные частоты (13) отсутствуют. Более того, эти частоты достаточно велики и достаточно хорошо разделены.

Неравенство

$$
\hbar_{\omega m n}^{0} / k T \ll 1
$$

выражает условие «высокотемпературного приближения». Оно в ЯМР всегда хорошо соблюдается, хотя обусловливает лишь небольшое упро-
щение кинетики.

С упе ропе р а то р релаксаци и. По требованию (5) б должен преобразовывать эрмитовый оператор $P(t)$ опять же в эрмитовый. Для этого необходимо и достаточно, чтобы между матричными элементами

$$
G_{m n, k l}=\left(\circlearrowleft A_{k l}, A_{m n}\right)
$$

существовали зависимости

$$
G_{n m, l k}=\left(G_{m n, k l}\right)^{*} .
$$

В частности, матричные элементы

$$
\Gamma_{k m}=G_{k h, m m}
$$

должны быть действительными числами.

Полезно представить пространство $\mathbf{O}$ в виде прямой суммы

$$
\mathbf{O}=\mathbf{O}_{A}+\mathbf{O}_{\perp} \text {. }
$$

$n$-мерное. подпространство $\mathbf{O}_{A}$ натянуто на $A_{m m}$, а $\mathbf{O}_{\perp}$, соответственно, на $A_{m n}, m \neq n$. В силу условий симметрии (8) супероператор релаксации распада-
ется по тем же подпространствам

$$
\text { (G) }=\text { (ு) }_{A}+\mathscr{B S}_{\perp} \text {. }
$$

При этом в клеточной матрице $(21) \mathfrak{g}_{\perp} \ldots$ диагональная подматрица.

В блоковской теории $\mathscr{\sigma}_{\perp}$ к тому же эрмитовый супероператор:

$$
\left(\text { (夕) }_{\perp}\right) *=\left(3_{\perp}\right. \text {. }
$$

Поэтому, учитывая (18), имеем

$$
G_{m n, m n}=G_{n m, n m}=-1 / \tau_{m n}
$$

в качестве единственных неисчезающих элементов матрицы $\mathfrak{S}_{\perp}$. В силу условия (7) введенное в (23) время поперечной релаксации $\tau_{m n}$ перехода $m \rightarrow n$ оказывается положительным (см. ниже).

Специальным результатом блоковской теории, не вытекающим из требований (5)-(10), является следующая зависимость величин (23) от матричных элементов (19): 


$$
1 / \tau_{m n}=(1 / 2)\left(\Gamma_{m n}+\Gamma_{n m}\right)+\sum_{k \neq m, n}\left(\Gamma_{k m}+\Gamma_{k n}\right) .
$$

Действующая только в подпространстве $\mathbf{O}_{\boldsymbol{A}}$ подматрица $\boldsymbol{\varpi}_{\boldsymbol{A}}$ состоит из действительных чисел (19) - из вероятностей релаксационных переходов $m \rightarrow k$.

Ниже показывается, что следствием условия (6) является

$$
\Gamma_{m m}=-\sum_{k \neq m} \Gamma_{k m},
$$

а следствием условий (9) и (10) -

$$
\Gamma_{m k} / \Gamma_{k m}=\exp \left(\hbar \omega_{k m}^{0} / k T\right) .
$$

По (26) величины $\Gamma_{m k}, \Gamma_{k m}$ должны иметь одинаковый знак. В силу условия (7)

$$
\Gamma_{m k}>0 .
$$

В общем случае супероператор

$$
\mathbb{B}_{A} \in \operatorname{gl}(n, R)
$$

обладает как симметрической, так и кососимметрической составляющей. В частности, преобразование

$$
\begin{gathered}
\mathscr{\circlearrowleft}_{A} E=\sum_{k} \gamma_{k} A_{k k}, \\
\gamma_{k}=\sum_{m} \Gamma_{k m}=\sum_{m \neq k}\left(\Gamma_{k m}-\Gamma_{m k}\right)
\end{gathered}
$$

определяется только кососимметрической составляющей $\mathfrak{F}_{A}$.

Нетрудно убедиться, что следствиями уравнения (25) являются:

$$
\begin{gathered}
\operatorname{tr}\left(\left(5 A_{m m}\right)=0,\right. \\
\operatorname{det}(5 A=0 .
\end{gathered}
$$

Стало быть, $\mathscr{G}_{A}$ проектирует в $(n-1)$-мерное подпространство $\mathbf{O}_{A}{ }^{0}$, ортогональное к $E$ и содержащее операторы с нулевым следом.

Подматрицу супероператора $\mathfrak{G}_{A}$, действующую только в $\mathbf{O}_{A}{ }^{0}$, будем обозначать через $\mathscr{G}_{A}^{0}$. В приближении $(16) \Gamma_{m k} \approx \Gamma_{k m}$ и

$$
\left(\mathscr{S}_{A}^{0}\right)+=\mathbb{S}_{A}^{0},
$$

но (5) $_{A}$ в целом должен содержать кососимметрическую часть. Необходимость в этом возникает при введении равновесного состояния (9), отличного (хотя и мало) от $(1 / n) E$.

Благодаря свойству (32) решение уравнения

$$
\mathscr{6}_{A} P^{0}=0
$$

существует. Оно единственно, если только

$$
\operatorname{det}\left(\mathbb{S}_{A^{\prime}}^{0} \neq 0\right. \text {. }
$$

Вследствие соотношений (25) выраженное на языке $A$-базиса уравнение (34) принимает вид уравнения детального баланса. Оно имеет решение

$$
\tilde{\pi}_{m}^{n} / \tilde{\pi}_{k}^{0}=\Gamma_{m k} / \Gamma_{k m},
$$


совпадающее с больцмановским распределением только в силу (26). Введем в подпространство $\mathbf{O}_{A}$ новый ортогональный базис $J_{j}$

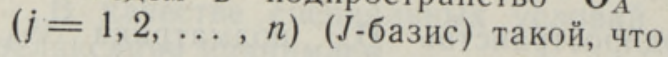

$$
\begin{gathered}
J_{1}=(1 / n) E, \\
\left(J_{j}, J_{k}\right)=(1 / n) \delta_{j k} .
\end{gathered}
$$

Тогда $\mathbf{O}_{A}^{0}$ будет натянуто на $J_{j}(j>1)$, а первый ряд матрицы $\mathfrak{G}_{A}$ (на том же базисе) будет состоять из нулей. Пусть

$$
\mathscr{\mathscr { S }}_{A} J_{1}=\sum_{j=2}^{n} \delta_{j} J_{j}
$$

Принимая во внимание свойство (33), выберем $J$-базис таким, чтобы

$$
\left(3_{A}^{0} J_{j}=-\left(1 / \tau_{j}\right) J_{j}\right. \text {. }
$$

В силу (7) времена продольной релаксации $\tau_{j}(j=2,3, \ldots, n)$ будут положительными (см. ниже). Если на языке $J$-базиса

$$
P^{0}=J_{1}+\sum_{j=2}^{n} \pi_{j}^{0} J_{j},
$$

то по уравнениям (34), (39)

$$
\delta_{j}=\pi_{j}^{0} / \tau_{j}
$$

Подпространство

$$
\mathbf{O}^{0}=\mathbf{O}_{A}^{0}+\mathbf{O}_{\perp}
$$

инвариантно относительно $\mathfrak{g}(t)+\mathfrak{G}$. Супероператор

$$
\mathrm{CS}^{0}=\mathrm{CS}_{A}^{0}+\mathrm{CS}_{\perp}
$$

действует только в $\mathbf{O}^{0}$. Согласно изложенному выше,

$$
\begin{gathered}
\left(\mathfrak{S}^{0}\right)^{+}=\mathscr{B}^{0}, \\
(\mathfrak{S}(t))^{+}=-\mathfrak{G}(t) .
\end{gathered}
$$

Подведем некоторые итоги. Определенные условия опыта описываются гамильтонианом (3), температурой $T$ и матрицей $\mathfrak{G}_{A}$. Располагая этими данными, можно построить управляющий движением супероператор $\mathfrak{g}(t)+(\mathfrak{s}$. За исключением правил $(22),(24)$, способ построения (5) однозначно вытекает из постулатов $(5)-(10)$.

\section{Основные свойства уравнения Блока}

Л ин ей н ость. Общее решение линейного уравнения (1) компактно выражается с помощью зависящего от времени супероператора (про-
пагатора) $\mathfrak{L}(t, 0)\left[{ }^{6}\right]$ :

$$
P(t)=\mathfrak{R}(t, 0) P(0) .
$$

Пропагатор подчиняется уравнению движения 


$$
\frac{d \mathfrak{Q}(t, 0)}{d t}=(\mathfrak{S}(t)+(\mathfrak{S}) \mathfrak{Q}(t, 0)
$$

и начальному условию

$$
\mathfrak{L}(0,0)=\Subset,
$$

где Е - единичный супероператор.

Зависимость движения $P(t)$ от $P(0)$ отражена в пропагаторе. Поэтому проблема предсказания движения сводится к установлению взаимно-однозначного соответствия

$$
\mathfrak{g}(t)+\mathfrak{G} \leftrightarrow \mathfrak{2}(t, 0) .
$$

При этом требования (5)-(10) устанавливают определенные ограничения на выбор величин, входящих в соответствие (50).

Так как $P(t)$ эрмитовый оператор, то должно быть верно не только (5), но и

$$
\mathfrak{Q}(t, 0) \in \mathbf{G} \mathbf{L}\left(n^{2}, R\right) .
$$

Это приводит к зависимости типа (18) между матричными элементами супероператоров $\mathfrak{S}(t)$, ऊ, $\mathfrak{2}(t, 0)$.

Чтобы выяснить ограничения, налагаемые требованием (6), разложим $P(t)$ на $A$-базисе и составим уравнение

$$
\frac{d}{d t}(\operatorname{tr} P)=0
$$

Поскольку уравнение (52) должно соблюдаться при любом $P(t)$, то

$$
\left(\left(B A_{k l}, E\right)=0\right. \text {. }
$$

Частным видом уравнения (53) являются соотношения (25).

Итак, (5) и $\mathfrak{\mathcal { G }}(t)$ проектируют в $\mathbf{O}^{0}$, являющееся, тем самым, инвариантным подпространством движения. Зависящая от времени часть $P(t)$ управляется действующим только в $\mathbf{O}^{0}$ пропагатором $\mathfrak{2}^{0}(t, 0)$. Уравнением движения этого пропагатора является

$$
\frac{d \mathbb{2}^{0}(t, 0)}{d t}=\left(\mathfrak{S}^{(}(t)+\mathbb{S}^{0}\right) \mathfrak{2}^{0}(t, 0) \text {. }
$$

Чтобы расширить $\mathfrak{2}^{0}(t, 0)$ до $\mathfrak{\Omega}(t, 0)$, из матричных элементов

$$
\lambda_{j k}(t, 0)=\left(\mathfrak{L}(t, 0) J_{k}, J_{j}\right)
$$

следует дополнительно указать элементы

$$
\lambda_{1 k}(t, 0)=\delta_{1 k}
$$

и элементы $\lambda_{j 1}(t, 0)$. Последние нужны для описания траектории

$$
\mathfrak{L}(t, 0) J_{1}=J_{1}+\sum_{j=2}^{n} \lambda_{j 1}(t, 0) J_{j} .
$$

Эти матричные элементы подчиняются своей особой, неоднородной, системе уравнений:

$$
\frac{d \lambda_{j 1}(t, 0)}{d t}=\delta_{j}+\sum_{k=2}^{n}\left\{\mathfrak{S}_{E}(t)_{j k}-\frac{1}{\tau_{k}} \delta_{j k}\right\} \lambda_{k 1}(t, 0) .
$$


Включив в уравнения $(57),(58)$ только члены $j, k=2,3, \ldots, n$, мы учли тем самым требования (8)-(10).

Если некоторые супероператоры $\mathfrak{2}, \mathfrak{R}$ обладают свойством (56), то тем же свойством обладает произведение $\mathfrak{2 N}$, но не $\mathfrak{z}^{-1}$. Поэтому допустимые пропагаторы $\mathfrak{2}(t, 0)$ ограничены пределами определенной полугруппы, входящей в состав $\mathrm{GL}\left(n^{2}, R\right)$ (см. ниже).

Н е о б р а ти м ость. Пусть $P_{s}(t)$ - некоторая траектория из семейства (47). Введем оператор девиации

$$
\Delta(t)=P(t)-P_{s}(t) \in \mathbf{O}^{0} .
$$

На основе вышеизложенного

$$
\begin{aligned}
& \Delta(t)=\Omega^{0}(t, 0) \Delta(0), \\
& \frac{d \Delta}{d t}=\left(\mathscr{Y}(t)+\left(\mathcal{S}^{0}\right) \Delta .\right.
\end{aligned}
$$

В силу свойств супероператора $\mathscr{\S}(t)$ скорость изменения

$$
\frac{d}{d t}(\Delta, \Delta)=2\left(\otimes^{0} \Delta, \Delta\right) \leqslant 0
$$

зависит только от $5^{\circ}$ и имеет постоянный знак. Чтобы требование (7) о конечности длин векторов выполнялось, этот знак должен быть отрицательным, а все $\tau_{j}>0, \tau_{m n}>0$. Знак нуль в неравенстве (62) относится к случаю $P(t)=P_{s}(t)$. Стало быть,

$$
\Delta(t) \rightarrow 0 \text { при } t \rightarrow \infty .
$$

Семейство (47) постепенно сжимается к одной траектории $P_{s}(t)$. В случае релаксации полезно выбирать $P_{s}(t)=P^{0}$. Тогда (63) даст приближение к равновесному состоянию $P^{0}$. Ясно, что таки'х состояний может быть только одно. Поэтому должны соблюдаться уравнения (32), (35).

На языке пропагаторов свойство необратимости (63) гласит:

$$
\begin{aligned}
& \Omega^{0}(t, 0) \rightarrow 0, \\
& \mathfrak{L}(t, 0) J_{1} \rightarrow P_{s}(t) .
\end{aligned}
$$

Все пропагаторы $2\left(t_{2}, t_{1}\right)\left[{ }^{6}\right]$ со свойством $t_{1} \leqslant t_{2}$ принадлежат к полугруппе допустимых пропагаторов, определяемых по

$$
0<\operatorname{det} \mathfrak{\imath}\left(t_{2}, t_{1}\right) \leqslant 1 \text {. }
$$

\section{ЛИТЕРАТУ РА}

1. B l o ch, F., Phys. Rev., 102, № 1, 104-135 (1956).

2. Бл ом бе рген Н., Нелинейная оптика, М., «Мир», 1966.

3. Ф а й н В. М., Х а н и н Я. И., Квантовая радиефизика, М., «Сов. радио», 1965.

4. Бе р тен Ф., Основы квантовой электроники, М., «Мир», 1971.

5. С и н и в э э В., Изв. АН ЭССР, Физ. Матем., 29, № 3, 288-295 (1980).

6. С ин и в е э В., Изв. АН ЭССР, Физ. Матем., 24, № 1, 35-48 (1975).

7. B in ch, G., Mol. Phys., 15, № 5, 469-480 (1968).

8. H ubbard, P. S., Rev. Mod. Phys., 33, № 2, 249-264 (1961).

Институт химической $u$
биологической физики
Академии наук Эстонской ССР
Поступила в редакцию 9/II 1980 


\section{SINIVEE}

\section{BLOCHI KVANTKINEETILISTE VORRANDITE ANALUUS}

Uurimuses on lineaarteisenduste keelt kasutades kirjeldatud n-nivoolise spinnsüsteemi Blochi võrrandite üldisi omadusi.

\section{SINIVEE}

\section{ANALYSIS OF THE BLOCH MASTER EQUATION}

General solutions of the Bloch master equation of a $n$-level spin system can be described in terms of time-dependent linear superoperators $\mathfrak{L}(t, 0)$ as shown by Eq. (47). The propagator $\mathfrak{L}(t, 0)$ belongs to the group $\mathbf{G L}\left(n^{2}, R\right)$, whereas its infinitesimal superoperator $\mathfrak{S}(t)+\mathbb{W}$ is governed by the corresponding Lie algebra $\mathrm{gl}\left(n^{2}, R\right)$. If $P(t)$ is considered as a vector of the $n^{2}$-dimensional space $\mathbf{O}$ of linear operators, the antihermitean superoperator (2) is defined by the spin Hamiltonian (3), whereas the linear superoperator $\mathbb{E}$ describes interactions with the thermal bath. A list of requirements (5) - (10) can be set up, which determine the actual shape of $\mathfrak{G}$ and of the Bloch equations up to the relationships (22), (24). This list includes general properties (5) (7) of the density operator, a symmetry property (8) of $\mathfrak{G}$ as well as the Boltzman Law (9), (10) for the equilibrium state $P^{0}$. It turns out that the propagator belongs to a semigroup of $\mathbf{G L}\left(n^{2}, R\right)$ defined by the condition that the $\left(n^{2}-1\right)$-dimensional subspace $\mathbf{O}^{0}$ of traceless operators must be an invariant subspace of $\mathfrak{L}(t, 0)$. $\mathscr{G}^{0}$ is a hermitean linear superoperator acting in $\mathbf{O}^{0}$. $\mathfrak{G}$ projects into $\mathbf{O}^{0}$. The propagator induces rotations and contractions of the space $\mathbf{O}^{0}$. As a consequence, the whole family of trajectories contracts into one trajectory - into the steady state. 